\title{
Haploids and doubled haploids in Brassica spp. for genetic and genomic research
}

\author{
Alison M. R. Ferrie • Christian Möllers
}

Received: 4 June 2010/Accepted: 25 August 2010/Published online: 14 September 2010

(C) Her Majesty the Queen in Right of Canada 2010

\begin{abstract}
The availability of a highly efficient and reliable microspore culture protocol for many Brassica species makes this system useful for studying basic and applied research questions. Microspores and microspore-derived embryos are ideal targets for modification by mutagenesis and transformation. Regenerated doubled haploid plants are widely used in breeding programs and in genetic studies. Furthermore, the Brassica microspore culture system allows the identification of genomic regions and genes involved in the microspore embryogenic response, spontaneous diploidization and direct embryo to plant conversion. This review summarizes current achievements and discusses future perspectives.
\end{abstract}

Keywords Doubled haploids · Breeding · Transformation · Mutagenesis · Genomics

\section{Introduction}

Haploids and doubled haploids have been used for practical and basic research for many years. In early studies, the objective of producing a haploid plant was to develop a doubled haploid (DH); a homozygous, true-breeding line to be used in a plant breeding program, and numerous reviews

\section{A. M. R. Ferrie ( $\square)$}

Plant Biotechnology Institute, National Research Council, 110 Gymnasium Place, Saskatoon, SK S7N 0W9, Canada e-mail: Alison.Ferrie@nrc-cnrc.gc.ca

\section{Möllers}

Department für Nutzpflanzenwissenschaften, Abteilung Pflanzenzüchtung, Georg-August-Universität Göttingen, Von Siebold Str. 8, 37075 Göttingen, Germany

e-mail: cmoelle2@gwdg.de have been written on this topic (Thomas et al. 2003; Forster and Thomas 2005; Szarejko and Forster 2007). DH lines are valued for use in breeding programs because traits can be fixed without multiple generations of back-crossing. DH lines are also useful in hybrid breeding and in the development of mapping populations.

The true magnitude of the importance of this technology in basic research was not realized until researchers had access to a highly efficient doubled haploidy system and started manipulating microspores and the resulting doubled haploid plants in mutagenesis, transformation, genetics, and genomics studies. The development of a routine, consistent, highly efficient doubled haploidy protocol is integral to utilizing these technologies. As mentioned in a previous review in this issue (Ferrie and Caswell 2010), a number of factors influence microspore embryogenesis. These include genotype, donor plant conditions, developmental stage of the microspore, pretreatments, media constituents, and culture environment. These factors have to be optimized for each species and genotype prior to exploitation of the microspores. Microspore cultures are ideal for manipulation as a large population of haploid single cells can be easily isolated. The main advantage of such cells as targets for studies is that a change can be made to a microspore at the haploid level, a plant regenerated, and chromosomes doubled resulting in a truebreeding plant which carries the modification made at the haploid level.

During the last few years, there has been a revival of haploidy technology research and subsequently, a number of publications on the application of doubled haploids in genetics and genomics. While there is an abundance of papers on different plant species, we are focusing this review on Brassica species. Highly efficient model systems for microspore embryogenesis and subsequent $\mathrm{DH}$ plant 
regeneration have been developed with a number of Brassica species (Custers 2003; da Silva Dias 2003; Ferrie 2003; Hansen 2003). Microspore culture systems are available for all Brassica species within U's triangle and application of this technology has been published (Ferrie and Keller 2004; Friedt and Zarhloul 2005). Tremendous progress has been achieved and microspore culture technology and its applications are being used in breeding programs around the world to develop improved cultivars and to answer fundamental biological questions.

\section{Utilization of doubled haploids in breeding, mutagenesis and transformation}

\section{Utilization of doubled haploids in breeding}

Application of doubled haploid technology to breeding Brassica crops is limited mainly by three factors: first, the embryogenic potential of the microspores, secondly, the capacity of the microspores to diploidize with or without treatment with anti-microtubule agents like colchicine and lastly, the capability for direct embryo to plant conversion (Möllers and Iqbal 2009). Although methods have been optimized to achieve the maximum response, it is clear that there exist significant genetic differences between genotypes (Barro and Martín 1999; Ferrie et al. 1995; Kuginuki et al. 1999). Thereby, the number of genes involved in the expression of each of those three traits and their mode of inheritance is probably quite different (Siebel and Pauls 1989; Zhang and Takahata 2001). A relevant question for applying doubled haploid technology in breeding is to know whether the positive alleles involved in all three critical steps mentioned above are preferentially inherited to the next generation, i.e. that plants regenerated from microspore culture do show an improved regeneration capacity when they are used again as donor plants for microspore culture. The selection of a line with enhanced embryogenesis capability has been identified in $B$. rapa (Ferrie et al. 1995) and in B. napus, as the highly embryogenic line Topas DH4079 is a selection from the cultivar Topas (W. A. Keller, personal communication).

The creation of haploid and doubled haploid plants was first envisioned to assist plant breeding programs. The development of a homozygous line in one generation rather than several generations is beneficial as it accelerates the breeding of desirable varieties. It can take up to 10 years to develop a cultivar in a conventional breeding program for annual self-pollinating crops, but with the use of DH lines, this can be reduced by 3-4 years. Even with 6 years of self pollination in a conventional plant breeding program, one can only achieve about $98 \%$ homozygosity, compared to $100 \%$ with doubled haploidy techniques. With cross- pollinating crops, the DH plants can be used as parental inbreds in crosses or for the production of hybrid varieties. There are a number of additional advantages to using doubled haploidy protocols in breeding programs, including the ability to fix important traits, identify dominant and recessive genes, and work with a smaller population of plants. A relatively new breeding method utilizing DHs is reverse breeding. This crop improvement approach allows the breeder to produce parental lines from any heterozygous plant (Dirks et al. 2009). However, it may not always be beneficial when using doubled haploidy methods in a plant breeding program, as pointed out in a recent review (Pink et al. 2008). It includes one round of recombination, which may be an advantage or disadvantage as beneficial gene combinations are maintained but detrimental linkages are not broken. As noted before, DH techniques are genotype and species dependent and therefore are not available for all species or all genotypes within a species. For species in which routine DH protocols are not available, the cost of implementing a DH program and initiating research in this area may be too costly for some breeding programs.

In 2003, Thomas et al. (2003) listed over $200 \mathrm{DH}$ commercial cultivars from 12 crop species. Of those species, 49 cultivars were B. napus and two cultivars were $B$. juncea. This list is now outdated and incomplete as breeding organizations are retaining cultivar development information as proprietary. Most of the currently grown Canadian canola (B. napus) varieties are derived from DH technology and many of the $B$. oleracea vegetable breeding programs also use doubled haploidy (M. Farnham, Personal Communication).

\section{Utilization of doubled haploids in mutagenesis}

Conventional mutagenesis, wherein the seeds are exposed to a mutagenic agent, has been used for many years to generate variation. This technique has been successful in the development of new cultivars (Maluszynski et al. 2000), but conventional mutagenesis protocols are timeconsuming in regards to detection and evaluation of the mutants. As the seed embryo is multicellular, the inherent risks with mutagenesis include the production of chimeras or the loss of a desired trait. Haploid microspores are an ideal system for conducting mutagenesis studies as millions of microspores can be isolated and cultured in a small space such as a petri plate. Microspores surviving the mutagenesis treatment can be regenerated into plants and then selection for desired traits can be conducted. Any DNA change made at the haploid single cell stage will be homozygous in the doubled haploid plant facilitating identification of recessive and dominant mutants. On the other hand, recessive mutations with deleterious effects at 
the homozygous stage cannot be recovered using this method.

A prerequisite for any mutagenesis study is a highly efficient microspore culture system, and therefore Brassica species are ideal candidates. There have been a number of studies using Brassica microspore culture to generate lines with altered fatty acid profiles or changes in agronomic characteristics. Microspore mutagenesis protocols using EMS (ethylmethane sulfonate) were developed for B. napus, B. juncea, and B. rapa (Ferrie et al. 2008) and B. nigra (Ferrie, unpublished). Over 80,000 Brassica haploid/doubled haploid plants were produced, and field evaluation and fatty acid analysis were conducted on a number of the lines. For B. rapa, there were differences found among the lines in the oleic acid, linolenic acid and saturated fatty acid levels. Differences were also observed among the lines of B. napus and B. juncea (Ferrie et al. 2008).

Ultraviolet light has also been used as a mutagenic agent (Barro et al. 2002; Sonntag and Rudloff 2004; Beaith et al. 2005). B. napus doubled haploid plants regenerated from microspores exposed to UV light exhibited changes in the level of saturated fatty acids. Differences in erucic acid levels and glucosinolate levels of $B$. carinata DH lines were reported after the microspores had been subjected to UV light or EMS (Barro et al. 2001, 2002). Valuable agronomic characteristics have also been observed in DH plants regenerated from microspores exposed to UV (McClinchey and Kott 2008). In vitro selection was used in combination with UV mutagenesis to identify mutants with enhanced cold tolerance. The mutant lines were subjected to freezer tests and field testing to determine the level of cold tolerance. Several lines showed enhanced tolerance with one line advancing to yield trials (McClinchey and Kott 2008). The combination of EMS mutagenesis and in vitro selection has been used in a novel approach in order to produce lines resistant to Sclerotinia sclerotiorum (Liu et al. 2005). Microspore culture was carried out on $B$. napus and the resulting haploid seedlings were allowed to produce callus. This callus was subjected to EMS followed by culture on different concentrations of oxalic acid, the toxin produced by the pathogen. Two DH lines were identified as having increased tolerance to $S$. sclerotiorum compared to the parental lines based on greenhouse experiments and field trials.

The timing of the mutagenic treatment of microspores is critical, as treatment should take place prior to or just after the first mitotic cell division and before any potential spontaneous chromosome doubling. Dosage is also important, as microspores are more sensitive than other tissue or seeds. The type of mutagenic agent is also a factor to consider when mutagenizing microspores. Chemical and physical mutagens have been used successfully with
Brassica microspores, including EMS (ethylmethane sulfonate), ENU (ethyl nitrosourea), $\mathrm{NaN}_{3}$ (Sodium azide), MNU ( $N$-methyl- $N$-nitrosourea), gamma rays, $\mathrm{X}$-rays, and UV (ultraviolet) (See Szarejko and Forster 2007 for review). He et al. (2007) treated both microspores and microspore-derived embryos to $\mathrm{UV}, \mathrm{NaN}_{3}$, or EMS. With $\mathrm{UV}$, there was a decrease in embryogenesis with an increase in UV levels, and embryo germination decreased. When $\mathrm{NaN}_{3}$ and EMS were applied to the microspores, there was an increase in embryo yield at low concentrations of the mutagen but not at the higher concentrations nor with the longer exposure times. Enhanced embryogenesis and plant regeneration were observed with the treatment of $\mathrm{NaN}_{3}$.

In vitro selection studies have also been carried out alone or in conjunction with microspore mutagenesis programs. Most of the early work was done with herbicide tolerance in Brassica. The active ingredient of the herbicide was included in the culture medium as a selection agent or as the mutagen (Beversdorf and Kott 1987; Zhang and Takahata 1999). In vitro selection for freezing tolerance was attempted with winter oilseed rape (Janska et al. 2010). Microspores which survived a selection pressure of tran-4-hydroxy-L-proline (Hyp) and were able to develop into embryos were regenerated to plants. Of the resulting $12 \mathrm{DH}$ lines, three had higher levels of proline in their leaves and increased freezing tolerance and two had increased proline levels but lower freezing tolerance. In vitro selection has also been used to enhance oil quality traits. Lines with different levels of erucic acid (Albrecht et al. 1995) or oleic acid (Möllers et al. 2000) have been identified.

\section{Utilization of doubled haploids in transformation}

Transformation is a useful tool for crop improvement and basic research. Microspores, microspore-derived embryos, and doubled haploid plants have been used as the starting material for transformation experiments. As with mutagenesis, microspores are advantageous for transformation because of the single cell, haploid nature of the microspore that is able to regenerate into an embryo and subsequently produce a homozygous true-breeding plant carrying the gene of interest. A highly efficient doubled haploidy protocol is required for any transformation protocols, making Brassica species ideal candidates.

Different microspore transformation methods have been attempted in Brassica including Agrobacterium tumefaciens (Pechan 1989; Dormann et al. 2001), particle bombardment (Fukuoka et al. 1998; Nehlin et al. 2000), microinjection (Jones-Villeneuve et al. 1995), and electroporation (Guerche et al. 1987; Jardinaud et al. 1993). A recent paper combined particle bombardment and Agrobacterium-mediated 
transformation in B. napus microspores (Abdollahi et al. 2009a). The integrated system of bombarding DNA coated particles followed by Agrobacterium was more effective with regards to generating GUS positive microspores, however, no embryos were produced. The authors did determine that the density of the microspores in culture, the gold particle size, and the addition of osmotic agents were important for enhancing transformation efficiency. Transformation efficiency was based on the number of GUSpositive cells. A recent publication on a novel transformation system in triticale involved the use of cell-penetrating peptides (Chugh et al. 2009), which have the capability to translocate across cell membranes, and have been used in mammalian systems to move molecules into the cell. It is thought that peptide uptake occurs through pores in the cell membrane. Microspores are different from other cells due to the thick microspore exine that creates a barrier for uptake of the DNA. In order to enhance microspore transformation, some researchers have used enzymes or other methods to reduce or puncture the exine.

Microspore-derived embryos (MDE) have also been used as the initial material for transformation using microinjection (Neuhaus et al. 1987; Swanson and Erickson 1989; Huang 1992) or a combination of microprojectile bombardment and DNA imbibition (Chen and Beversdorf 1994). MDE of winter rapeseed have been transformed by Agrobacterium tumefaciens (CegielskaTaras et al. 2008). The transgenic winter oilseed rapeseed plant (DH T-39) proved to be homozygous for the introduced trait. Considering the growth habit of winter rapeseed, development of a homozygous transgenic line in one generation will greatly expedite the breeding process. Transformation of MDE with biolistics in combination with secondary embryogenesis has been reported recently (Abdollahi et al. 2009b, 2010). The hypocotyls of MDE were bombarded and, since the hypocotyl is amenable to secondary embryogenesis, there is a potential that the secondary embryos would be transformed. The transformed secondary embryos were regenerated to plants and analyzed molecularly and biochemically for the introduced genes. Transgenic plants of B. napus cultivars Global and $\mathrm{PF}_{704}$ were regenerated (Abdollahi 2010) at a high frequency.

Transformation and DH technology have also been used to create homozygous lines with enhanced tolerance to clubroot (Reiss et al. 2009) or pollen beetle (Ahman et al. 2006). Transgenic lines were developed first and then doubled haploid methodology was used in order to generate lines homozygous for the trait of interest, thereby speeding up the breeding process. Combining microspore culture technology with the newer techniques such as the use of zinc fingers for precise gene targeting may also speed up the breeding process.

\section{Identification of genomic regions and genes related to microspore embryogenesis}

Mapping genes related to microspore embryogenesis

Tremendous differences among B. napus genotypes in their embryogenic response of cultured microspores have been reported (Ferrie 2003; Ferrie and Keller 2007). However, the genetic basis of the genotypic differences in microspore culture response remains largely unknown. The approaches pursued to unravel the phenomenon of "microspore embryogenesis" can be divided into those investigating the inheritance of the genes determining the embryogenic potential and those comparing gene expression profiles of induced and non-induced microspores of genotypes with contrasting embryogenic response. The first approach involves using heterozygous $\mathrm{F} 1$ plants derived from crosses between two different genotypes and the second approach usually is performed with homozygous lines.

Regarding the first approach, results from early work with isozymes and molecular markers frequently showed distorted marker segregation in DH populations. Foisset et al. (1993) showed for five out of ten isozyme loci studied, a significant distorted segregation in microspore derived DH populations in two out of five crosses, whereas no distorted segregation was observed in F2 plants obtained from the same F1 plants.

Zhang and Takahata (2001) performed diallel crosses between four cultivars of $B$. napus and B. campestris with differential embryogenic response. The results suggested that in B. napus embryogenesis is mainly controlled by gene loci with additive effects. The embryogenic response of F2 plants $(n=80)$ derived from the cross Lisandra (responsive) $\times$ Kamikita (non-responsive) indicated segregation of two genes controlling embryogenesis. In a follow up study, Zhang et al. (2003) identified RAPD markers linked to microspore embryogenic potential. In oilseed rape, from 52 markers showing polymorphisms, the segregation of 14 marker alleles (27\%) was distorted. Of these markers, 11 showed a significant excess of Lisandra alleles (the responsive parent), and 3 showed a significant excess of Kamikita alleles. With 8 randomly selected markers out of those 14 a segregation analysis was performed in a F2 population. Six markers showed the expected Mendelian segregation in the F2 population, indicating that they may be linked to genes relevant for microspore embryogenesis. However, by comparing embryo yield and segregation data, only 3 of those 6 markers were identified to be related to microspore embryogenic ability. The plants with Lisandra alleles produced significantly higher embryo yields than plants with Kamikita alleles. In a similar study, Tanhuanpää et al. (1994) found distorted segregation in a DH population 
derived from a cross between Topas and a low linolenic acid mutant line for 5 RAPD markers. The distortion was in favour of the responsive parent Topas.

Comparing the $\mathrm{F} 2$ and two $\mathrm{DH}$ populations derived from a cross between the microspore culture-responsive cultivar Topas and the non-responsive cultivar Westar, Cloutier et al. (1995) found that the DH populations showed a significantly higher molecular marker distortion towards the alleles of the responsive cultivar Topas, which was not the case in the F2 population. Assuming that plants derived from a cross between a responsive and a non-responsive parent accumulate favourable alleles from the responsive parent (Wan et al. 1992), DNA markers linked to those alleles will display an excess of alleles from the responsive parent in the microspore derived populations but will show a mendelian segregation in the F2 population. Cloutier et al. (1995) found that markers positioned on linkage groups 1 and 18 showed significant segregation distortion towards Topas alleles in both microspore-derived populations and segregated according to the expected Mendelian ratios in the F2 population. Hence, linkage groups LG1 and LG18 likely carry chromosomal regions which are associated with microspore culture responsiveness. In a similar preliminary study, Ajisaka et al. (1999) compared segregation of RAPD marker alleles in a DH population and an F2 population derived from a cross between the highly responsive Chinese cabbage line, Homei 09 and the nonresponsive turnip line, Siloga S2. They observed distorted marker allele segregation in favour of the Homei 09 alleles on six linkage groups, whereas on two linkage groups, markers were found that segregated in favour of Siloga S2. However, F2 plants carrying putative positive alleles from Homei 09 did not always show superior response in microspore culture.

Ferreira et al. (1994) compared segregation ratios of RFLP loci in a DH and a F2 population obtained from the same F1 plant of a cross between the winter cultivar Major and a DH line of the spring cultivar Stellar. More linkage groups showing markers with distorted segregation were found for the DH population and there were almost no overlap of markers showing distorted segregation in both populations. The strongest distorted segregations were found for two marker loci on LG19 and one marker loci on LG20, favouring the alleles of DH line Stellar and Major, respectively.

A hindrance for comparisons between different populations is that an assignment of linkage groups of above mentioned molecular marker maps to linkage groups of current populations following the $\mathrm{N}$-nomenclature (Parkin et al. 1995) or the international A1-A10, C1-C9-nomenclature (http://www. brassica.info/resource/maps/lg-assignments.php) is impossible. However, there are a number of more recently developed molecular marker maps of $\mathrm{DH}$ populations that use either one of those two nomenclatures and hence allow comparison of linkage groups (Table 1).

Uzunova (1994) found in a DH population derived from the cross Mansholts $\times$ Samourai, distorted marker segregation $(P=0.05$ and $P=0.01)$ for ten linkage groups, from which 6 were in favour of the Mansholts alleles and 4 in favour of the Samourai alleles (Table 1). Following the above mentioned hypothesis, this could indicate that Mansholts has a better microspore embryogenic potential than Samourai, but that Samourai, on four linkage groups, also carries some alleles which support microspore embryogenesis. However, the strongest distorted segregations were found for marker alleles on linkage group N17 (LG7; Uzunova 1994), with segregation ratios of up to 48:122, favouring the Samourai alleles. The effect of these N7 loci on microspore embryogenesis may outbalance the effects of the other loci favouring the Mansholts alleles. No marker alleles with distorted segregation were found on linkage groups N1, N3, N11, N12, N14, N15, N16, N18 and N19.

In DH populations derived from the crosses Darmor $x$ Yudal, Darmor $\times$ Samourai, and Stellar $\times$ Drakkar, 25, 14 and $9 \%$ of the mapped markers showed skewed segregation, respectively (Lombard and Delourme 2001; Delourme et al. 2006). In Darmor $\times$ Yudal, clusters with distorted marker alleles were found on many linkage groups (Table 1). In the DH populations derived from Darmor $x$ Samourai and Stellar $\times$ Drakkar, comparatively fewer linkage groups with distorted marker alleles were detected. With one exception (Darmor $\times$ Yudal: N7/Y-8, N7/D-1), all distorted marker alleles together on one linkage group favoured either one of the parents. Linkage group N13 (717) showed skewed segregation in three populations, but the skewed region on N13 of Stellar $\times$ Drakkar did not correspond exactly to those on Darmor $\times$ Yudal or Darmor $\times$ Samourai (Lombard and Delourme 2001). In the DH populations Darmor $\times$ Yudal and Darmor $\times$ Samourai, the same regions on linkage groups N3 and N7 carried loci showing distorted marker allele segregation. Foisset et al. (1996) reported that the linkage group DY4 (N3) could correspond to a region involved in microspore-culture responsiveness identified by Cloutier et al. (1995). In this region, segregation bias is towards the responsive parental lines Darmor-bzh and Darmor in both DH populations, which reinforces the hypothesis that those marker alleles are linked to loci relevant to embryogenic potential. They also discussed that distorted segregation around a locus on linkage group DY6 (N6) may be due to meiotic disturbances/gametic selection because RFLP markers were observed to be biased in a $\mathrm{F} 2$ population as well as in a $\mathrm{DH}$ population from the cross Westar $\times$ Topas (Landry et al. 1991; Cloutier et al. 1995). In another DH population derived from a cross between two winter oilseed rape 
Table 1 Distorted marker segregation observed in DH populations

\begin{tabular}{|c|c|c|}
\hline Cross & LG*/favouring alleles of number of markers & Reference \\
\hline $\begin{array}{l}\text { DH Mansholts }(\mathrm{M}) \times \\
\text { DH Samourai }(\mathrm{S})\end{array}$ & $\begin{array}{l}\text { N2/M-8, N4/M-3, N5/M-1, N6/M-11, N7/S-6, N8/S-2, N9/S-2, } \\
\text { N10/M-4, N13/M-7, N17/S-8, }\end{array}$ & Uzunova $(1994)^{+}$ \\
\hline Darmor $(\mathrm{D}) \times$ Yudal $(\mathrm{Y})$ & $\begin{array}{l}\text { N1/D-1, N2/Y-12, N3/D-15, N5/Y-5, N6/Y-26, N7/Y-8, N7/D-1, N9/D-17, } \\
\text { N11/D-3, N12/D-4, N13/Y-14, N14/D-1, N15/D-3, N16/D-15, N19/Y-13, }\end{array}$ & $\begin{array}{l}\text { Lombard and } \\
\text { Delourme }(2001)^{\$}\end{array}$ \\
\hline Darmor (D) $\times$ Samourai (S) & N1/D-1, N3/D-16, N7/S-5, N8/D-4, N11/S-2, N13/S-13 & $\begin{array}{l}\text { Lombard and } \\
\text { Delourme }(2001)^{\$}\end{array}$ \\
\hline Stellar $(\mathrm{S}) \times$ Drakkar $(\mathrm{D})$ & N1/S-1, N3/D-1, N4/S-1, N5/D-8, N9/S-2, N13/S-11, N14/S-3 & $\begin{array}{l}\text { Lombard and } \\
\text { Delourme }(2001)^{\$}\end{array}$ \\
\hline Sollux $(\mathrm{S}) \times$ Gaoyou $(\mathrm{G})$ & $\begin{array}{l}\text { N1/G-14, N2/G-19, N3/G-10, N5/S-19, N6/S-2, N6/G-1, N7/S-13, } \\
\text { N9/S-32, N10/G-20, N11/S-22, N11/G-3, N12/S-18, N13/G-16, } \\
\text { N14/S-3, N14/G-1, N15/S-4, N17/S-10, N18/S-15, N19/S-4 }\end{array}$ & Zhao et al. $(2005)^{\S}$ \\
\hline Rapid (R) × NSL96/25 (N) & N7/R, N11/R, N12/R & $\begin{array}{l}\text { Delourme et al. } \\
\text { (2006) }\end{array}$ \\
\hline Express (E) × R53 (R) & $\begin{array}{l}\text { N1/R-1, N2/R-6, N3/E-10, N4/R-6, N5/R-1, N6/R-2, N7/E-7, N8/R-8, } \\
\text { N9/E-9, N10/R-3, N11/E-3, N12/E-3, N13/R-1, N14/R-4, N15/E-15, N18/R-1, } \\
\text { N19/E-17 }\end{array}$ & Radoev (2007) \\
\hline $\begin{array}{l}\text { Quantum }(\mathrm{Q}) \times \text { Resyn } \\
\text { No. } 2127-17(\mathrm{R})\end{array}$ & $\begin{array}{l}\text { N1/Q-17, N2/Q-8, N3/R-11, N4/Q-1, N5/Q-14, N5/R-1, N7/Q-10, N8/Q-4; } \\
\text { N9/R-2, N11/Q-7, N12/Q-15, N13/Q-8, N13/R-10, N14/R-1, N15/Q-6, } \\
\text { N16/R-1, N16/Q-1, N18/Q-10, N19/Q-4 }\end{array}$ & Chen et al. $(2007)^{\S \S}$ \\
\hline Early Big $(\mathrm{E}) \times$ TO1000DH3 $(\mathrm{T})$ & N11/E, N12/E, N13/T, N14/E, N15/E, N16/T, N17/E, N18/T & $\begin{array}{l}\text { Iniguez-Luy et al. } \\
\text { (2009) }\end{array}$ \\
\hline \multicolumn{3}{|c|}{ * Linkage group N-nomenclature according to Parkin et al. (1995) } \\
\hline \multicolumn{3}{|c|}{+ Linkage group assignment to N-nomenclature according to Wolfgang Ecke, personal communication } \\
\hline \multicolumn{3}{|c|}{ \$ Linkage group assignment to N-nomenclature according to Delourme et al. (2006) } \\
\hline \multicolumn{3}{|c|}{$\S$ Based on an extended linkage map with 525 markers; personal communication Jianyi Zhao } \\
\hline
\end{tabular}

genotypes with differing oil content (Rapid $\times$ NSL96/25), only three linkage groups with marker loci showing distorted segregation favouring the Rapid alleles were detected (Table 1; Delourme et al. 2006).

In a $\mathrm{DH}$ population derived from the cross Sollux $\times$ Gaoyou, $48 \%$ of the 525 mapped markers showed a significant $(P=0.01)$ deviation from the expected 1:1 segregation ratio, with 144 and 110 being skewed towards Sollux and Gaoyou, respectively (Zhao et al. 2005; Jianyi Zhao personal communication). The distorted markers on a linkage group were generally clustered (not shown) and together they were in favour of either one parent, e.g. distorted marker alleles on N1, N2, and N3 were in favour of the Gaoyou alleles and distorted marker alleles on N5 and N9 were in favour of the Sollux alleles (Table 1). Altogether, distorted markers on 10 linkage groups were in favour of the Sollux alleles and distorted markers on 9 linkage groups were in favour of the Gaoyou alleles. Only linkage groups N6, N11 and N14 had marker alleles in favour of both parents. However, single or two linked marker alleles with distorted segregation on linkage groups N6 and N14 are more likely to occur due to scoring errors or misclassification of marker bands. Linkage groups N4,
N8 and N16 did not show any marker alleles with distorted segregation.

In another DH population derived from the cross of winter oilseed rape cultivar Express with the resynthesized line R53, 31.4\% of the mapped markers exhibited a significant $(P=0.05)$ deviation from the expected 1:1 segregation ratio (Radoev 2007). An excess of Express alleles was observed for 79 markers $(69.3 \%)$, whereas R53 alleles prevailed for the other 35 markers $(30.7 \%)$. Markers with distorted segregation were found on all linkage groups, N16 and N17. Again, most of the distorted marker alleles were clustered and those located together on a linkage group were in favour of either parent. None of the linkage groups carried distorted marker alleles in favour of both parents (Table 1).

For a DH population derived from the cross Quantum $\times$ resynthesized line No.2127-17, Chen et al. (2007) described a high proportion of loci showing segregation distortion: 70 SSR loci (17.6\%) and 59 SRAP loci (14.8\%) showed distorted segregation ratio $(P=0.01)$, respectively. Most of loci with distorted segregation (103 loci) were skewed towards the Quantum parent (Table 1), indicating that Quantum may have a superior embryogenic 
response. Interestingly, Quantum is a microspore derived DH Canadian cultivar (Stringam et al. 1995), which may have inherited an improved microspore culture response.

Udall et al. (2005) reported that in four B. napus DH populations between 26 and $41 \%$ of the loci showed a significant distorted segregation $(P=0.01)$. Furthermore, considerable differences in the strength of distortion, i.e. the significance level ranging from $P=0.05$ to $P=0.001$ were found. Unfortunately, linkage groups and loci showing distorted allele segregation were not shown.

In a $B$. oleracea $\mathrm{DH}$ mapping population created from a cross of a DH broccoli line (Early Big) and a DH rapid cycling (TO1000DH3) line, Iniguez-Luy et al. (2009) found segregation distortion of alleles at 139 loci. Most of the loci exhibiting excess of Early Big alleles (77 loci) were clustered on LGs C1 (N11), C2 (N12), C4 (N14), C5 (N15) and C7 (N17); those with an excess of TO1000DH3 alleles (62 loci) were on LGs C3 (N13) (in its entirety), C6 (N16) and C8 (N18) (Table 1). For further discussion of distorted marker allele segregation in B. oleracea see Pink et al. (2008).

In summary, the information provided in Table 1 show that on all linkage groups of the oilseed rape genome regions involved in microspore embryogenic response have been identified. However, some linkage groups have been identified as relevant more frequently than others and show a larger number of distorted markers (Table 2). For example, linkage group N13 was found in all 7 considered populations to carry distorted marker loci, whereas linkage groups N16 and N17 carry distorted marker loci in only two populations. However, those results are influenced by the size of the linkage group (for example: linkage group N13 is one the largest linkage groups).

Presently, it is only a hypothesis that distorted marker allele segregation is caused by their linkage to genes related to the embryogenic potential of microspores. Skewed marker allele segregation may also occur because

Table 2 Number of DH populations (N) showing distorted segregation on individual linkage groups (LG) (Results from Table 1, except results from populations Rapid $\times$ NSL96/25 and Early Big $\times$ TO1000DH3)

\begin{tabular}{lllllllll}
\hline LG & N & $\begin{array}{l}\text { Sum of } \\
\text { markers }\end{array}$ & LG & N & $\begin{array}{l}\text { Sum of } \\
\text { markers }\end{array}$ & LG & N & $\begin{array}{l}\text { Sum of } \\
\text { markers }\end{array}$ \\
\hline N1 & 6 & 35 & N8 & 4 & 18 & N15 & 4 & 28 \\
N2 & 5 & 53 & N9 & 6 & 64 & N16 & 2 & 17 \\
N3 & 6 & 63 & N10 & 3 & 27 & N17 & 2 & 18 \\
N4 & 4 & 11 & N11 & 5 & 40 & N18 & 3 & 26 \\
N5 & 6 & 49 & N12 & 4 & 40 & N19 & 4 & 38 \\
N6 & 4 & 42 & N13 & 7 & 80 & & & \\
N7 & 6 & 50 & N14 & 5 & 13 & & & \\
\hline
\end{tabular}

of linkage to genes relevant for colchicine induced diploidization or efficient direct embryo to plant conversion. In normal experiments, embryos not converting to plants are excluded from further analysis. To distinguish at which level the distortion occurs, marker analysis would need to be done at the MDE level and subsequently, embryo mortality, diploidization, and plantlet regeneration rates need to be recorded. Although DNA amounts sufficient for PCR analysis can be extracted from a single MDE cotyledon (Nath et al. 2007), this has so far not been used for studying marker allele segregation.

Furthermore, skewed marker allele segregation may not be caused by factors related to embryogenesis of the unicellular microspore, but may be caused by genetic factors relevant to earlier stages of the meiosis. If gamete production in meiosis is already affected, then distorted marker allele segregation should become evident in F2 and in DH populations equally. However, F2 populations have only been analysed in a few cases parallel to DH populations. So far, the hypothesis of a gametic selection in some of the biased regions cannot be excluded, as suggested already by Ferreira et al. (1994) and Foisset et al. (1996).

To predict which of the parents of the above mentioned crosses may have a better embryogenic potential is difficult. The total number of linkage groups with marker alleles favouring either one of the two parents is not a good indicator, because nothing is known about the size of the effects they may have on embryogenesis. In general, one would assume that the stronger the distortion of the marker alleles, the more pronounced the effect of the linked gene(s) on embryogenesis. Unfortunately, only a few of the above published papers indicate the strength of the marker allele distortion. Significant marker allele segregation may also occur by chance and hence, may not appear if a second DH population would be generated from the same cross (cf. Cloutier et al. 1995). Again, the stronger the distortion of the marker alleles, the less likely the distortion is caused by chance. Larger groups of marker alleles showing distorted segregation occur less likely by chance, whereas smaller groups with only one or few distorted marker alleles may simply be due to chance or marker scoring errors. To overcome these problems, microspore culture experiments need to be repeated two or three times and independently generated DH populations from the same cross need to be studied for consistency of distorted marker allele segregation.

Alternative approaches for mapping genes related to microspore embryogenesis

Apart from the work of Ajisaka et al. (1999), no research in Brassica species was continued beyond reporting distorted segregations in doubled haploid populations. Mapping of genes influencing microspore embryogenesis in classical 
DH populations is difficult, because reliable scoring of the trait 'microspore embryogenic response' in populations of about $150 \mathrm{DH}$ lines is not only a tremendous task, but also error-prone, because donor-plant constitution as well as microspore developmental stage in relation to bud size may vary considerably. Furthermore, results from mapping a trait in a DH population that shows distorted segregation not only for this trait but also for associated molecular markers are questionable (for discussion, see Foisset et al. 1996). If the hypothesis "marker loci showing distorted segregation in $\mathrm{DH}$ populations are linked to genes for microspore embryogenesis (excluding gamete selection)" is valid, then $\mathrm{DH}$ lines showing a combination of positive alleles from both parents should show superior microspore embryogenesis compared to DH lines derived from the same cross and not having those positive alleles. This could be tested with a limited number of DH lines. More recently developed intervarietal substitution lines (Howell et al. 1996; Burns et al. 2003, Wolfgang Ecke, personal communication) provide a means to more precisely evaluating the effect of certain genomic regions on microspore embryogenesis. Preferentially, lines should be analysed that carry genomic regions which previously have been shown to cause distorted segregation in DH populations (cf. Table 1). This approach has the advantage that at a given time only a limited number of genotypes need to be compared (lines with a certain substituted genomic region vs. lines without that genomic region) and that homozygous intervarietal substitution lines can be propagated via seeds, i.e. replicated microspore culture experiments can be performed.

Segregating F2 populations provide a possibility to circumvent problems related to distorted segregation, but only individual F2 plants are available for phenotyping, unless F2 plants are clonally propagated. Alternatively, recombinant inbred populations may be developed (Butruille et al. 1999; Fu et al. 2007). Together, these approaches share the disadvantage that large numbers of plants need to be characterized for their embryogenic response and that the detected variation is limited to the variation derived from the two parental lines. Association mapping (Hasan et al. 2008; Ecke et al. 2010) provides another possibility for evaluating the variation for microspore embryogenesis in a larger germplasm collection bypassing problems related to distorted segregation.

Gene expression studies of embryogenic microspores

Comparative analysis of gene expression in induced embryogenic microspores versus gene expression in noninduced non-embryogenic microspores of the same genotype provides insight into which genes are related to microspore embryogenesis. Following this approach, a number of genes specific for microspore embryogenesis have been identified in B. napus (Boutilier et al. 2002; Malik et al. 2007; Hosp et al. 2007; Tsuwamoto et al. 2007; Tsuwamoto and Takahata 2008; Malik and Krochko 2009). Malik et al. (2007) identified 16 genes from which expression profiles were informative and predictive for embryogenesis in microspore cultures. From those 16 genes, five genes (BnFUS3, BnLEC2, BnLEC1, BnUP1, BnUP2) were only transcribed during the induction and the development of microspore embryos and development of zygotic seed. Six genes (BnNAPIN, BnBBM1, BnFAD1, BnWOX9, BnABI3, BnATS1) showed a similar expression pattern, except that some of these also showed expression in some other tissue, e.g. BnBBM1 and BnFAD1 showed strong expression in roots. Five genes (BnLRR1, BnCP1, BnCYP78A, BnWOX2, BnCYP81F) were expressed in developing embryos and also in other sporophytic tissues, but nonetheless reliably marked the transition in microspores from the gametophytic (pollen) developmental pathway to the sporophytic (embryogenic) pathway. Interestingly, seven of the marker genes for microspore embryogenesis are known to be transcription factors (LEC1, LEC2, FUS3, ABI3, BBM1, WOX2, WOX9). For in depth discussions of the functions of those 16 genes, see Malik et al. (2007). The expression levels of BnLEC1, BnLEC2, BnBBM1, and BnUP1 in cultured microspores of highly responsive Topas-DH4079 and poorly responsive cultivar Allons, Westar, and Garrison at 3 and/or 7 days of culture were strongly correlated with their embryogenic potential (Malik et al. 2007). Furthermore, ectopic expression of LEC1, LEC2, or BBM1 was shown to be sufficient to induce embryogenesis in somatic tissues and more recent studies have demonstrated that LEC1, LEC2, and FUS3 are required for zygotic and somatic embryogenesis in Arabidopsis (Malik et al. 2007, and references therein).

From the poorly embryogenic cultivar Westar, four DH lines could be regenerated under specific conditions. Those lines showed to have acquired an improved embryogenic potential which was inherited to the next generation (Malik et al. 2008). One of those lines, DH2, was compared in more detail to Westar. Transcription analysis showed that BnLEC1 expression in Westar microspore cultures was markedly delayed at day 1 and day 3 as compared to DH2 cultures. Expression of the other marker genes, namely BnLEC2, BnABI3, BnBBM1, BnUP1, and BnWOX9, was uniformly reduced at all time points in Westar cultures as compared with DH2 cultures (Malik et al. 2008). In contrast, transcripts for pollen-expressed genes were predominant in the recalcitrant Westar microspores. Furthermore, microarray analyses of gene expression using a $B$. napus seed cDNA array revealed a large number (117) of differentially expressed genes between 7 days old heat-treated microspores of Westar and derived DH2 (Malik et al. 
2008). Remaining heterozygosity in the microspore donor plant material and somaclonal variation may have contributed to the observed gross pleiotropic changes in gene expression. Notably, besides being embryogenic, DH-2 plants showed some clear alterations in morphology and architecture as compared to Westar.

By combined transcriptome and proteome analysis, Joosen et al. (2007) identified approximately 220 genes and 32 proteins that are expressed between the 2 and 4 cell and globular/heart stages of embryo development. Altogether, quite a number of candidate genes for microspore embryogenesis have been identified in B. napus (see also Xiang et al. 2008; Whittle et al. 2010).

\section{Future perspectives}

Since the first report of in vitro production of doubled haploid plants in the 1960s, there has been much improvement in protocol development, efficiency, and application of the doubled haploidy method. Recently there has been renewed interest in the use of $\mathrm{DH}$ for new breeding methods, transformation, and genomics research. Much of this focus has been with the highly efficient microspore culture systems, which have resulted in the advancement of determining the molecular aspects of microspore embryogenesis.

Circumstantial evidence for the influence of certain regions of the $B$. napus genome on microspore embryogenesis has been obtained from molecular marker maps developed using DH populations. Numbering of the linkage groups according to the nomenclature of Parkin et al. (1995) or the international standard has allowed comparison of linkage groups of different DH populations. However, clearly more locus specific markers need to be mapped in different populations to allow for a better alignment of the linkage groups across several DH populations. Only then, a meta-analysis can be performed and genomic regions causing distorted marker segregation in several DH populations can be identified more precisely. The use of F2 and recombinant inbred populations for studying inheritance of microspore embryogenesis response bypasses the problem of distorted trait and marker segregation and allows identification of marker loci linked to genes relevant for gametic selection. Future research will also show if epigenetic phenomena play a role in microspore embryogenesis. However, the analysis of epigenetic control of gene expression in male and female gametogenesis has just started (Takeda and Paszkowski 2006; Sheldon et al. 2008; Le Trionnaire and Twell 2010).

Comparative analysis of gene expression has led to the discovery of a number of genes that are specifically up regulated in induced embryogenic microspores. Although in some cases ectopic expression of these genes in transgenic plants proved their role in embryogenesis, this can only be taken as evidence to explain natural genetic variation observed for microspore embryogenesis. However, those genes are valid candidate genes for microspore embryogenesis and their mapping in the oilseed rape genome will show if their position overlaps with regions showing distorted marker segregation. Progress in mapping those genes may be hampered by the presence of multiple gene copies in amphidiploid oilseed rape. On the other hand, the whole genome sequences of B. rapa and B. napus will be available soon, which will simplify identification of duplicated gene loci.

Precise identification of genomic regions relevant for microspore embryogenic response will also show if those regions are closely linked in coupling or repulsion to loci relevant for yield or oil content. It can be anticipated that cultivars and breeding lines that have been obtained via microspore culture have inherited an improved response with respect to microspore embryogenesis, colchicine induced chromosome doubling and MDE to plant conversion. Since application of the DH technology in oilseed rape breeding is steadily increasing, it can be expected that the tissue culture ability of current breeding material is increasing in parallel. Hence, molecular markers for tissue culture ability may not be required in a normal breeding program, but may be useful if non adapted material is used in crosses.

Acknowledgments We thank Wei Chen, Wolfgang Ecke and Jianyi Zhao for providing us unpublished results.

\section{References}

Abdollahi MR, Corral-Martinez P, Mousavi A, Salmanian AH, Moieni A, Segui-Simarro JM (2009a) An efficient method for transformation of pre-androgenic, isolated Brassica napus microspores involving microprojectile bombardment and Agrobacterium-mediated transformation. Acta Physiol Plant 31: 1313-1317

Abdollahi MR, Moieni A, Salmanian AH, Mousavi A (2009b) Secondary embryogenesis and transient expression of the $\beta$-glucuronidase gene in hypocotyls of rapeseed microsporederived embryos. Biol Plant 53:573-577

Abdollahi MR, Moieni A, Mousavi A, Salmanian AH (2010) High frequency production of rapeseed transgenic plants via combination of microprojectile bombardment and secondary embryogenesis of microspore-derived embryos. Mol Biol Rep. doi: 10.1007/s11033-010-0158-3

Ahman IM, Kazachkova NI, Kamnert IM, Hagberg PA, Dayteg CI, Eklund GM, Meijer LJO, Ekbom B (2006) Characterisation of transgenic oilseed rape expressing pea lectin in anthers for improved resistance to pollen beetle. Euphytica 151:321-330

Ajisaka H, Kuginuki Y, Shiratori M, Ishiguro K, Enomoto S, Hirai M (1999) Mapping loci affecting the cultural efficiency of microspore culture of Brassica rapa L. syn campestris L. using DNA polymorphism. Breed Sci 49:187-192 
Albrecht S, Möllers C, Röbbelen G (1995) Selection in vitro for erucic acid content in segregating populations of microsporederived embryoids of Brassica napus. Plant Breed 114:210-214

Barro F, Martín A (1999) Response of different genotypes of Brassica carinata to microspore culture. Plant Breed 118:79-81

Barro F, Fernández-Escobar J, De la Vega M, Martin A (2001) Doubled haploid lines of Brassica carinata with modified erucic acid content through mutagenesis by EMS treatment of isolated microspores. Plant Breed 120:262-264

Barro F, Fernández-Escobar J, De la Vega M, Martín A (2002) Modification of glucosinolate and erucic acid contents in doubled haploid lines of Brassica carinata by UV treatment of isolated microspores. Euphytica 129:1-6

Beaith ME, Fletcher RS, Kott LS (2005) Reduction of saturated fats by mutagenesis and heat selection in Brassica napus $\mathrm{L}$. Euphytica 144:1-9

Beversdorf WD, Kott LS (1987) An in vitro mutagenesis/selection system for Brassica napus. Iowa State J Res 61:435-443

Boutilier K, Remko O, Sharma VK, Kieft H, Ouellet T, Zhang L, Hattori J, Liu C-M, van Lammeren AAM, Miki BLA, Custers JBM, van Lookeren Campagne MM (2002) Ectopic expression of BABY BOOM triggers a conversion from vegetative to embryonic growth. Plant Cell 14:1737-1749

Burns MJ, Barnes SR, Bowman JG, Clarke MHE, Werner CP, Kearsey MJ (2003) QTL analysis of an intervarietal set of substitution lines in Brassica napus: (i) Seed oil content and fatty acid composition. Heredity 90:39-48

Butruille DV, Guries RP, Osborn TC (1999) Linkage analysis of molecular markers and quantitative trait loci in populations of inbred backcross lines of Brassica napus L. Genetics 153:949-964

Cegielska-Taras T, Pniewski T, Szala L (2008) Transformation of microspore-derived embryos of winter oilseed rape (Brassica napus L.) by using Agrobacterium tumefaciens. J Appl Genet 49:343-347

Chen JL, Beversdorf WD (1994) A combined use of microprojectile bombardment and DNA imbibition enhances transformation frequency of canola (Brassica napus L.). Theor Appl Genet 88:187-192

Chen W, Zhang Y, Liu X, Chen B, Tu J, Tingdong F (2007) Detection of QTL for six yield-related traits in oilseed rape (Brassica napus) using DH and immortalized F2 populations. Theor Appl Genet 115:849-858

Chugh A, Amundsen E, Eudes F (2009) Translocation of cellpenetrating peptides and delivery of their cargoes in triticale microspores. Plant Cell Rep 28:801-810

Cloutier S, Cappadocia M, Landry BS (1995) Study of microsporeculture responsiveness in oilseed rape (Brassica napus L.) by comparative mapping of a F2 population and two microsporederived populations. Theor Appl Genet 91:841-847

Custers JBM (2003) Microspore culture in rapeseed (Brassica napus L.). In: Maluszynski M, Kasha KJ, Forster BP, Szarejko I (eds) Doubled haploid production in crop plants. Kluwer, Dordrecht, pp 185-193

Da Silva Dias JC (2003) Protocol for broccoli microspore culture. In: Maluszynski M, Kasha KJ, Forster BP, Szarejko I (eds) Doubled haploid production in crop plants. Kluwer, Dordrecht, pp 195-204

Delourme R, Falentin C, Huteau V, Clouet V, Horvais R, Gandon B, Specel S, Hanneton L, Dheu JE, Deschamps M, Margale E, Vincourt P, Renard M (2006) Genetic control of oil content in oilseed rape (Brassica napus L.). Theor Appl Genet 113: $1331-1345$

Dirks R, van Dun K, de Snoo CB, van den Berg M, Lelivelt CL, Voermans W, Woudenberg L, de Wit JPC, Reinink K, Schut JW, vab der Zeeuw E, Vogelaar A, Freymark G, Gutteling EWKMN, van Drongelen P, Kieny M, Ellul P, Touraev A, Ma H, De Jong H, Wijnker E (2009) Reverse breeding: a novel breeding approach based on engineered meiosis. Plant Biotechnol J 7:837-845

Dormann M, Wang H-M, Oelck M (2001) Transformed embryogenic microspores for the generation of fertile homozygous plants. USA No. 6,316,694

Ecke W, Clemens R, Honsdorf N, Becker HC (2010) Extent and structure of linkage disequilibrium in canola quality winter rapeseed (Brassica napus L.). Theor Appl Genet 120:921-931

Ferreira ME, Williams PH, Osborn TC (1994) RFLP mapping of Brassica napus using doubled haploid lines. Theor Appl Genet 89:615-621

Ferrie AMR (2003) Microspore culture of Brassica species. In: Maluszynski M, Kasha KJ, Forster BP, Szarejko I (eds) Doubled haploid production in crop plants. Kluwer, Dordrecht, pp 195-204

Ferrie AMR, Caswell KL (2010) Isolated microspore culture techniques and recent progress for haploid and doubled haploid plant production. Plant Cell Tissue Organ Culture. doi: 10.1007/s11240-010-9800-y

Ferrie AMR, Keller WA (2007) Optimization of methods for using polyethylene glycol as a non-permeating osmoticum for the induction of microspore embryogenesis in the Brassicaceae. In Vitro Cell Dev Biol-Plant 43:348-355

Ferrie AMR, Palmer CE, Keller WA (1995) Haploid embryogenesis. In: Thorpe TA (ed) In vitro embryogenesis in plants. Kluwer Academic Publishers, Dordrecht, pp 309-344

Ferrie AMR, Taylor DC, MacKenzie SL, Rakow G, Raney JP, Keller WA (2008) Microspore mutagenesis of Brassica species for fatty acid modifications: a preliminary evaluation. Plant Breed 127:501-506

Foisset N, Delourme R, Lucas MO, Renard M (1993) Segregation analysis of isozyme markers on isolated microspore-derived embryos in Brassica napus L. Plant Breed 110:315-322

Foisset N, Delourme R, Barret P, Hubert N, Landry BS, Renard M (1996) Molecular-mapping analysis in Brassica napus using isozyme, RAPD and RFLP markers on a doubled-haploid progeny. Theor Appl Genet 93:1017-1025

Forster BP, Thomas WTB (2005) Doubled haploids in genetics and plant breeding. Plant Breed Rev 25:57-88

Friedt W, Zarhloul MK (2005) Haploids in the improvement of crucifers. In: Palmer CE, Keller WA, Kasha KJ (eds) Haploids in crop improvement II, biotechnology in agriculture and forestry, vol 56. pp 191-213

Fu FY, Liu LZ, Chai YR, Chen L, Yang T, Jin MY, Mar AF, Yan XY, Zhang ZS, Li JN (2007) Localization of QTLs for seed color using recombinant inbred lines of Brassica napus in different environments. Genome 50:840-854

Fukuoka H, Ogawa T, Matsuoka M, Ohkawa Y, Yano H (1998) Direct gene delivery into isolated microspores of rapeseed (Brassica napus L.) and the production of fertile transgenic plants. Plant Cell Rep 17:323-328

Guerche P, Charbonnier M, Jouanin L, Tourneur C, Paszkowski J, Pelletier G (1987) Direct gene transfer by electroporation in Brassica napus. Plant Sci 52:111-116

Hansen M (2003) Protocol for microspore culture in Brassica. In: Maluszynski M, Kasha KJ, Forster BP, Szarejko I (eds) Doubled haploid production in crop plants. Kluwer, Dordrecht, pp 217-222

Hasan M, Friedt W, Pons-Kühnemann J, Freitag NM, Link K, Snowdon RJ (2008) Association of gene-linked SSR markers to seed glucosinolate content in oilseed rape (Brassica napus ssp napus). Theor Appl Genet 116:1035-1049

He Y, Chen SB, Jin ZL, Wan GL, Xu L, Tang GX, Zhou WJ (2007) Effects of mutagenic treatments of isolated microspores and 
microspore-derived embryos on embryogenesis and plant regeneration in oilseed rape (Brassica napus L.). In: GCIRC Proceedings of the 12th international rapeseed congress, vol 2, pp 69-72, GCIRC, Wuhan, China

Hosp J, de Faria Maraschin S, Touraev A, Boutilier K (2007) Functional genomics of microspore embryogenesis. Euphytica 158:275-285

Howell PM, Lydiate DJ, Marshall DF (1996) Towards developing ISL in Brassica napus using marker-assisted selection. Genome 39:348-358

Huang B (1992) Genetic manipulation of microspores and microspore-derived embryos. In Vitro Cell Dev Biology-Plant 28: $53-58$

Iniguez-Luy FL, Lukens L, Farnham MW, Amasino RM, Osborn TC (2009) Development of public immortal mapping populations, molecular markers and linkage maps for rapid cycling Brassica rapa and $B$ oleracea. Theor Appl Genet 120:31-43

Janska A, Zelenkova S, Klima M, Vyvadilová M, Prasil IT (2010) Freezing tolerance and proline content of in vitro selected hydroxyproline resistant winter oilseed rape. Czech J Genet Plant Breed 46:35-40

Jardinaud M-F, Souvre A, Alibert G (1993) Transient GUS gene expression in Brassica napus electroporated microspores. Plant Sci 93:177-184

Jones-Villeneuve E, Huang B, Prudhomme I, Bird S, Kemble R, Hattori J, Miki B (1995) Assessment of microinjection for introducing DNA into uninuclear microspores of rapeseed. Plant Cell Tiss Organ Cult 40:97-100

Joosen R, Cordewener J, Supena EDJ, Vorst O, Lammers M, Maliepaard C, Zeilmaker T, Miki B, America T, Custers J, Boutilier K (2007) Combined transcriptome and proteome analysis identifies pathways and markers associated with the establishment of rapeseed microspore derived embryo development. Plant Physiol 144:155-172

Kuginuki Y, Miyajima T, Masuda H, Hida K, Hirai M (1999) Highly regenerative cultivars in microspore culture in Brassica oleracea L. var. capitata. Breed Sci 49:251-256

Landry BS, Hubert N, Etoh T, Harada JJ, Lincoln SE (1991) A genetic map for Brassica napus based on restriction fragment length polymorphism detected with expressed DNA sequences. Genome 34:543-552

Le Trionnaire G, Twell D (2010) Small RNAs in angiosperm gametophytes: from epigenetics to gamete development. Genes Dev 24:1081-1085

Liu S, Wang H, Zhang J, Fitt BDL, Xu Z, Evans N, Liu Y, Yang W, Guo X (2005) In vitro mutation and selection of doubled-haploid Brassica napus lines with improved resistance to Sclerotinia sclerotiorum. Plant Cell Rep 24:133-144

Lombard V, Delourme R (2001) A consensus linkage map for rapeseed (Brassica napus L.): construction and integration of three individual maps from DH populations. Theor Appl Genet 103:491-507

Malik MR, Krochko JE (2009) Gene expression profiling of microspore embryogenesis in Brassica napus. In: Touraev A, Forster BP, Jain SM (eds) Advances in haploid production in higher plants. Springer, Heidelberg, pp 115-125

Malik MR, Wang F, Dirpaul JM, Zhou N, Polowick PL, Ferrie AMR, Krochko JE (2007) Transcript profiling and identification of molecular markers for early microspore embryogenesis in Brassica napus. Plant Physiol 144:134-154

Malik MR, Wang F, Dirpaul JM, Zhou N, Hammerlindl J, Keller W, Abrams SR, Ferrie AMR, Krochko JE (2008) Isolation of an embryogenic line from non-embryogenic Brassica napus cv Westar through microspore embryogenesis. J Exp Bot 59:2857-2873
Maluszynski M, Nichterlein K, Van Zanten L, Ahloowalia BS (2000) Officially released mutant varieties-the FAO/IAEA database. Mutation Breed 12:1-84

McClinchey SL, Kott LS (2008) Production of mutants with high cold tolerance in spring canola. Euphytica 162:51-67

Möllers C, Iqbal MCM (2009) Doubled haploids in breeding winter oilseed rape. In: Touraev A, Forster BP, Jain SM (eds) Advances in haploid production in higher plants. Springer, Heidelberg, pp 161-170

Möllers C, Rücker B, Stelling D, Schierholt A (2000) In vitro selection for oleic and linoleic acid content in segregating populations of microspore derived embryos of Brassica napus. Euphytica 112:195-201

Nath UK, Iqbal MCM, Möllers C (2007) Early, non-destructive selection of microspore derived embryo genotypes in oilseed rape (Brassica napus L.) by molecular markers and oil quality analysis. Mol Breed 19:285-289

Nehlin L, Möllers C, Bergman P, Glimelius K (2000) Transient ß-gus and GFP gene expression and viability analysis of microprojectile bombarded microspores of Brassica napus. J Plant Physiol 156:175-183

Neuhaus G, Spangenberg G, Mittelsten Scheid O, Schweiger H-G (1987) Transgenic rapeseed plants obtained by the microinjection of DNA into microspore-derived embryoids. Theor Appl Genet 75:30-36

Parkin IAP, Sharpe AG, Keith DJ, Lydiate DJ (1995) Identification of the A and C genomes of amphidiploid Brassica napus (oilseed rape). Genome 38:1122-1131

Pechan PM (1989) Successful cocultivation of Brassica napus microspores and proembryos with Agrobacterium. Plant Cell Rep 8:387-390

Pink D, Bailey L, McClement S, Hand P, Mathas E, BuchananWollaston V, Astley D, King G, Teakle G (2008) Double haploids, markers and QTL analysis in vegetable brassicas. Euphytica 164:509-514

Radoev M (2007) Genetic analysis of heterosis in rapeseed (B. napus L.) by QTL mapping. Dissertation, Georg-August-Universität Göttingen. http://webdoc.sub.gwdg.de/diss/2007/radoev/radoev.pdf

Reiss E, Schubert J, Scholze P, Kramer R, Sonntag K (2009) The barley thaumatin-like protein Hv-TLP8 enhances resistance of oilseed rape plants to Plasmodiophora brassicae. Plant Breeding 128:210-212

Sheldon CC, Hills MJ, Lister C, Dean C, Dennis ES, Peacock WJ (2008) Resetting of FLOWERING LOCUS C expression after epigenetic repression by vernalization. Proc Natl Acad Sci (USA) 105:2214-2219

Siebel J, Pauls KP (1989) A comparison of anther and microspore culture as a breeding tool in Brassica napus. Theor Appl Genet 87:473-479

Sonntag K, Rudloff E (2004) Microspore mutagenesis in transgenic oilseed rape for the modification of fatty-acid composition. Acta Universitatis Latviensis Biol 676:227-230

Stringam GR, Bansal VK, Thiagarajah MR, Degenhardt DF, Tewari JP (1995) Development of an agronomically superior blackleg resistant canola cultivar in Brassica napus L. using doubled haploidy. Can J Plant Sci 75:427-439

Swanson EB, Erickson LR (1989) Haploid transformation in Brassica napus using an octopine-producing strain of Agrobacterium tumefaciens. Theor Appl Genet 78:831-835

Szarejko I, Forster BP (2007) Doubled haploidy and induced mutation. Euphytica 130:359-370

Takeda S, Paszkowski J (2006) DNA methylation and epigenetic inheritance during plant gametogenesis. Chromosoma 115:27-35

Tanhuanpää PK, Vilkki JP, Vilkki HJ (1994) Segregation and linkage analysis of DNA markers in microspore derived and F2 
populations of oilseed rape (Brassica napus L.). Euphytica 74:59-65

Thomas WTB, Forster BP, Gertsson B (2003) Doubled haploids in breeding. In: Maluszynski M, Kasha KJ, Forster BP, Szarejko I (eds) Doubled haploid production in crop plants, a manual. Kluwer Academic Publishers, Dordrecht, pp 337-349

Tsuwamoto R, Takahata Y (2008) Identification of genes specifically expressed in androgenesis-derived embryo in rapeseed (Brassica napus L.). Breed Sci 58:251-259

Tsuwamoto R, Fukuoka H, Takahata Y (2007) Identification and characterization of genes expressed in early embryogenesis from microspores of Brassica napus. Planta 225:641-652

Udall JA, Quijada PA, Osborn TC (2005) Detection of chromosomal rearrangements derived from homoeologous recombination in four mapping populations of Brassica napus L. Genetics 169:967-979

Uzunova M (1994) Erstellung einer RFLP-Karte von Raps (Brassica napus L.) und Kartierung erster züchterisch wichtiger Gene. Dissertation Georg-August-Universität Göttingen, FB Agrarwissenschaften

Wan Y, Rocheford TR, Widholm JM (1992) RFLP analysis to identify putative chromosomal regions involved in the anther culture response and callus formation of maize. Theor Appl Genet 85:360-365

Whittle CA, Malik MR, Li R, Krochko JE (2010) Comparative transcript analyses of the ovule, microspore, and mature pollen in Brassica napus. Plant Mol Biol 72:279-299

Xiang D, Datla R, Li F, Cutler A, Malik M, Krochko J, Sharma N, Fobert P, Georgers F, Tsang E, Klassen D, Koh C, Deneault JS, Nantel A, Nowak J, Keller W, Bekkaoui F (2008) Development of a Brassica seed cDNA microarray. Genome 51:236-242

Zhang F-L, Takahata Y (1999) Microspore mutagenesis and in vitro selection for resistance to soft rot disease in Chinese cabbage (Brassica campestris L. ssp. pekinensis). Breed Sci 49:161-166

Zhang FL, Takahata Y (2001) Inheritance of microspore embryogenic ability in Brassica crops. Theor Appl Genet 103:254-258

Zhang FL, Aoki S, Takahata Y (2003) RAPD markers linked to microspore embryogenic ability in Brassica crops. Euphytica 131:207-213

Zhao J, Dimov Z, Becker HC, Ecke W, Möllers C (2005) Oil content in a European $x$ Chinese rapeseed population: QTL with additive and epistatic effects and their genotype-environment interactions. Crop Sci 45:51-59 